\title{
Precision and accuracy of oculocentric direction for targets of different luminances
}

\author{
HAROLD E. BEDELL, MURRAY H. JOHNSON, and RAPHAEL BARBEITO \\ College of Optometry, University of Houston, Houston, Texas
}

\begin{abstract}
The precision and accuracy of specifying oculocentric directions were assessed by successively partitioning an $8.3^{\circ}$ space in the right field into a series of perceptually equal fractional spaces and then matching each partitioning target's direction in the left field. Three observers performed this task for four target luminances, ranging from 0.04 to $43 \mathrm{~cd} / \mathrm{m}^{2}$. The results show that luminance has virtually no effect on either the precision or accuracy of spatial partitioning; essentially no effect was obtained even when, for one observer, target luminance was reduced to nearly the absolute threshold. We interpret these data in terms of oculocentric direction's role in mediating visual behaviors.
\end{abstract}

That visual functioning is reduced when illumination is reduced has been documented and quantified in over a century of scientific research. For example, resolution of fine detail and identification of form (Koenig, 1897, cited in Helmholtz, 1924/1925, 1925; Schlaer, 1937), discrimination of color (Weale, 1951), and detection of temporal and spatial luminance contrast (Kelly, 1961; van Nes \& Bouman, 1967) are all impaired substantially as the luminance of targets is decreased. A visual function reportedly not affected by luminance is the discrimination of a target's meridian in the visual field, which Leibowitz, Myers, and Grant (1955a, 1955b) found to be as good at the light detection threshold as at much higher luminances. Oculomotor functions such as fixation, smooth tracking, and optokinetic nystagmus have been reported also to survive substantial reductions of target luminance with little or no performance decrement (Boyce, 1967; Steinman, 1965; Valciukas, 1972; Winterson \& Steinman, 1978).

The significance of Leibowitz's findings is their implication that the mechanism(s) by which target meridian is determined differ fundamentally from mechanisms that process other aspects of visual stimuli, which depend importantly upon stimulus luminance. Because of this important implication for how different aspects of the visual scene may be processed, we sought to replicate and extend the results of the Leibowitz et al. studies, using a task complementary to theirs. Specifically, we investigated the effect of target luminance on observers' precision and accuracy in specifying the oculocentric direction of targets with respect to the fixation locus and other visual targets along a single (the horizontal) meridian of the field. Precision is defined as the fineness or sensitivity of direc-

This work was supported by National Eye Institute Research Grant EY 03694 to Merton C. Flom. We thank Stan Klein for the program used to psychometrically fit our data and Gale Pedersen for assistance in analyzing the results.

The authors' mailing address is: College of Optometry, University of Houston, University Park, Houston, TX 77004. tion judgments; imprecision is measured as the variability of these judgments. Accuracy refers to how closely judgments of direction agree with physical directions; inaccuracy is determined by the magnitude of observers' constant errors. In general, we find that visual directions of targets outside the fovea are specified as precisely and accurately at low luminances as at moderate photopic levels.

\section{METHOD}

Observers' precision and accuracy in specifying targets' oculocentric visual directions were assessed utilizing a version of the classical monocular spatial partitioning task (Helmholtz, 1925; Ogle, 1932). In our version of this task, a standard spatial extent $\left(8.3^{\circ}\right.$ in the right visual field) was successively bisected into a series of subjectively equal fractional spaces (Bedell \& Flom, 1983). Spaces were defined by bright vertical lines that were presented on the otherwise dark field of a Commodore 2001 video display and subtended $3.4^{\prime} \times 60^{\prime}$ of arc at the observer's $50-\mathrm{cm}$ viewing distance. One line, containing a $3.4^{\prime}$-arc gap in its center, served as the target for fixation. The observer first specified the visual direction of a line that appeared to be midway between this fixation target and the line $8.3^{\circ}$ in the right field, thereby bisecting this right-field space into two perceptually equal half-spaces. With this bisecting line serving as a new standard, the half-space nearer to fixation was itself bisected to determine the direction of a line perceptually $1 / 4$ of the way between the fixation target and the now-extinguished $8.3^{\circ}$ rightfield standard. Subsequent bisection of this $1 / 4$ space (again, with all extraneous lines extinguished) yielded a line whose direction marked a space perceptually equal to $1 / 8$ of the original standard space. In addition to these successive bisections (determining lines with directions defining $1 / 2,1 / 4$, and $1 / 8$ of the $8.3^{\circ}$ standard space), lines were found that perceptually defined $3 / 8$ and $3 / 4$ of the standard space. Lines with these directions were determined by bisecting the space between the lines marking $1 / 4$ and $1 / 2$ of the standard space and the space between the line marking $1 / 2$ of the standard space and the $8.3^{\circ}$ standard line. Once the standard space had been partitioned in the above manner, the direction of each right-field line was matched in the left field with a line that was perceived to be symmetrically located with respect to the fixation target.

On each trial, the observer was presented with a line within the space to be bisected or on the opposite side of the fixation target from the line whose direction was to be matched. The presented 
line remained visible until the observer signaled that it appeared rightward or leftward of the visual direction at which a bisection or match would occur. The direction of the target line presented on each trial depended on the observer's previous responses, according to a randomly interleaved double staircase (Cornsweet, 1962). Staircases reversed direction after a changed response category was maintained for two successively presented directions of the target line, a decision rule designed to distribute trials across the entire range of the psychometric function. Trials for each bisection or match were terminated after seven response reversals were accumulated for each staircase at the minimum stepsize of $3.4^{\prime}$ arc visual direction.

Each observer's precision in specifying visual direction was quantified according to the region of target directions over which he made mixed responses (rightward and leftward) during each bisection and match. The width (in minutes of arc visual direction) of this distribution of mixed, or uncertain, responses was specified in terms of the standard deviation of the cumulative Guassian function that best fit (using a maximum likelihood criterion) the distribution of "rightward" responses. Accuracy in specifying visual direction was quantified by comparing the direction of each subjective bisection or match (the $50 \%$ point of the fitted Gaussian) to the corresponding physical fraction of the original standard space.

This procedure of successively bisecting a right-field space into multiple equal-appearing intervals and matching each interval in the left field was repeated for four luminances of the target lines, ranging from 0.04 to $43 \mathrm{~cd} / \mathrm{m}^{2}$. Luminances were graded, in approximately $1 \log$ unit steps, using Tiffen neutral filters and were calibrated using a Spectra Pritchard photometer. All observers first performed spatial partitions for line targets at the highest luminance; thereafter, the order of target luminances was counterbalanced across observers. To minimize effects of fatigue, the observers were limited to completing spatial bisections and matches for a single target luminance on 1 day and, in addition, took breaks (without disrupting adaptation) as needed. Experimental sessions, which were conducted with room illumination extinguished, lasted approximately $11 / 2 \mathrm{~h}$. One observer (H.B.) also performed the series of spatial partitions with the luminance of each target line adjusted to be visible on about $90 \%$ of the trials (i.e., near the absolute luminance threshold). For this condition, appropriate combinations of neutral and Polaroid filters were used to maintain clear visibility of the fixation line (luminance about $0.5 \mathrm{log}$ units above foveal threshold) and to adjust the luminance of the standard and target lines to near threshold.

The three authors, aged 26 to 34 years, served as observers. H.B. and R.B. were experienced psychophysical observers and highly practiced at monocular spatial partitioning; M.J. was less experienced and, prior to data collection, received a practice session consisting of about 600 trials with line targets at the highest luminance. All observations were made with the right eye only and natural pupils. Only Observer H.B. had a significant refractive error $(-2.50 \mathrm{sph})$, correction for which was worn during the experiments.

\section{RESULTS}

\section{Effect of Target Location}

With increasing eccentricity from the fovea, targets' oculocentric directions were specified less precisely. For example, collapsed across subjects and luminances, targets approximately $1^{\circ}$ from the fovea $\left(1 / 8\right.$ of the $8.3^{\circ}$ standard space) were specified with a precision of between $1^{\prime}$ and $2^{\prime}$ of arc, whereas targets approximately $4^{\circ}$ from the fovea $(1 / 2$ of the standard space) were specified to about 7' of arc. These results are in agreement with previous studies which found that precision of spatial bisection, expressed in minutes of arc, approximates the ec- centricity of the bisecting target, expressed in degrees (Andrews \& Miller, 1978; Bedell, Barbeito, \& Aitsebaomo, 1984; Levi \& Klein, 1983; Stevens \& Ducasse, 1912).

When expressed as a percentage of target eccentricity, precision of specifying the direction of a bisecting target in the right field averaged $2 \%$ to $3 \%$ (Figure 1 ). Precision in matching directions in the left field was equally good for Observer H.B., was slightly worse (about 4\% of target eccentricity) for Observer R.B., and was substantially poorer (averaging about $6 \%$ of the matching target's eccentricity) for Observer M.J. In the right field, bisection of the spaces defined by targets marking $1 / 4$ and $1 / 2$ of the standard space (yielding a line that defined $3 / 8$ of the standard space) and by the target marking $1 / 2$ of the standard space and the standard at $8.3^{\circ}$ (yielding a line that defined $3 / 4$ of the standard space) tended to be the most precise. This result and the generally poorer precision in matching targets' directions in the left field ${ }^{1}$ suggest that, in addition to depending on the target's eccentricity, precision also depends on the extent of the visual space being partitioned.

As compared with physically equal fractions of the original $8.3^{\circ}$ standard space, each observer made constant errors that accumulated across successive partitionings (Figure 2). In general, Observer H.B. tended relatively to underestimate directions farther from fixation (by defining physically larger spaces farther from fixation than nearer to fixation), R.B. tended to underestimate left-field directions, and M.J. tended to underestimate directions nearer to fixation, especially in the left field. ${ }^{2}$ Accumulated across multiple bisection settings, the maximum constant error was an approximately $20 \%$ underestimation of left-field spaces near fixation for Observer M.J. (Figure 2C). These errors, which were accumulated across multiple bisection settings, were larger than the constant errors for each bisection or match considered alone, which produced median values near $2 \%$ for $\mathrm{Ob}$ servers H.B. and R.B. and near 5\% for Observer M.J.

\section{Effect of Target Luminance}

Neither the precision nor the accuracy of specifying target directions was influenced significantly by decreasing target luminance from 43 to $0.04 \mathrm{~cd} / \mathrm{m}^{2}$ (Figures 1 and 2). That luminance had essentially no effect was confirmed by analysis of variance [for precision $F(3,6)=1.36, p>$ .3 ; for accuracy, $F(3,6)=0.85, p>.5$ ]. In addition to substantiating the effects of target location on precision and accuracy of directionalization (described above), the analysis of variance also indicated that precision was significantly influenced by the interaction of luminance and target location $[\mathrm{F}(30,60)=1.75, \mathrm{p}<.05]$. Inspection of the data indicated that this significant luminance $\times$ location interaction was produced by two abnormally imprecise matches in the left field for Observer M.J. at the two highest luminances. Although we believe this result to be spurious, it is clear that neither precision nor accuracy worsen as target luminance is decreased. 

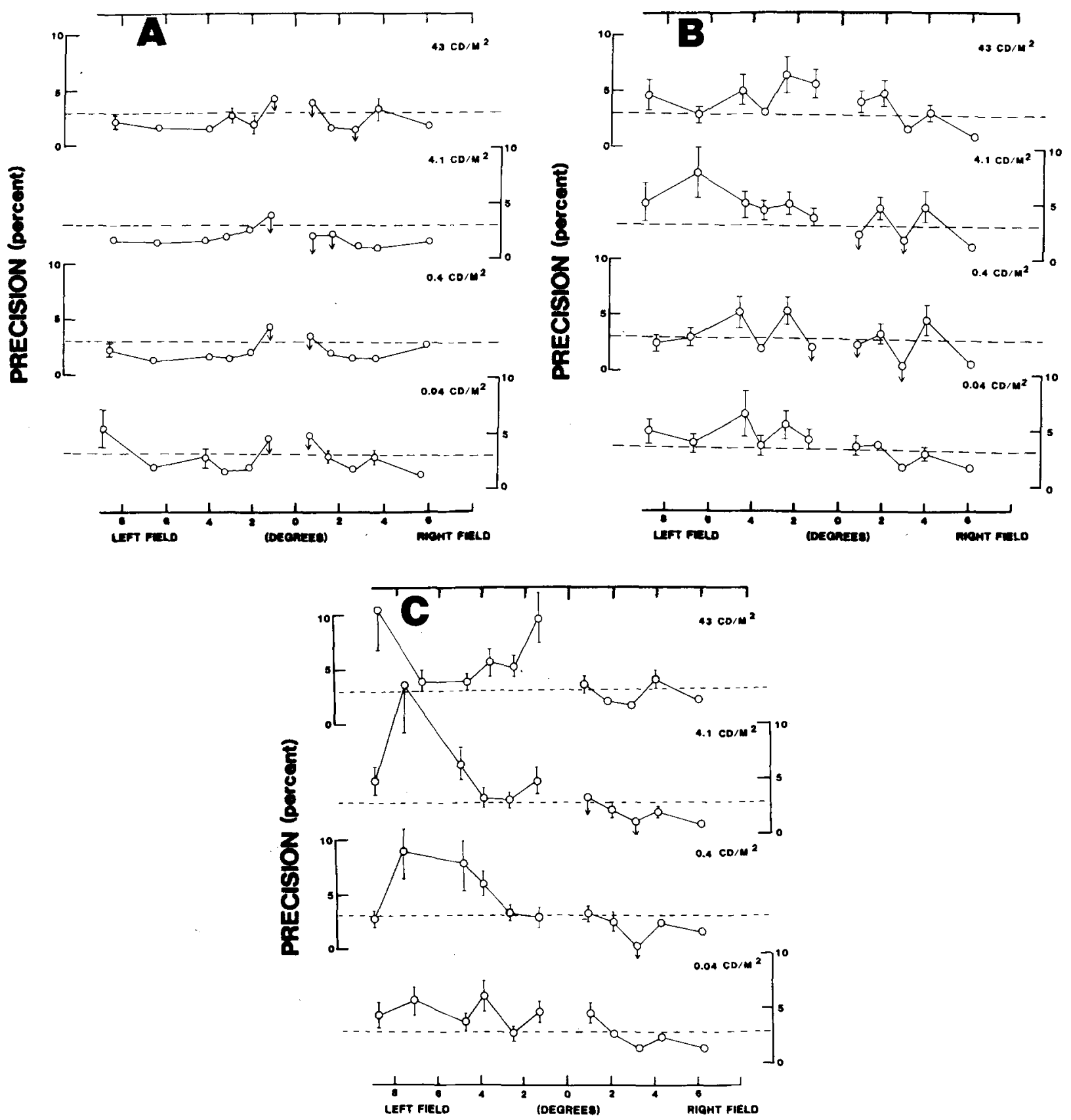

Figure 1. Precision of specifying oculocentric direction for targets at each of four luminances is plotted for three observers (H.B. in panel A, R.B. in panel B, and M.J. in panel C). Precision is plotted as a percentage of target eccentricity from fixation. Error bars represent \pm 1 standard error of estimate; downward arrows indicate that the psychometric function from which precision was determined was too steep to be fitted reliably and that the plotted point represents an upper limit.

Indeed, even when target luminances were reduced to nearly their absolute scotopic threshold, the precision and accuracy of Observer H.B.'s partition settings were affected very little (Table 1). The median value for precision of directionalization was $2.7 \%$ for targets near threshold and $2.0 \%$ for targets at the highest luminance. Consideration of the individual spatial bisections and matches revealed that, near threshold, 7 were less precise and 4 were more precise than at the highest luminance. In addition, Observer H.B. continued to underestimate directions farther from fixation when target luminance was near threshold, a pattern that was similar to that of the constant errors he made at higher luminances. 

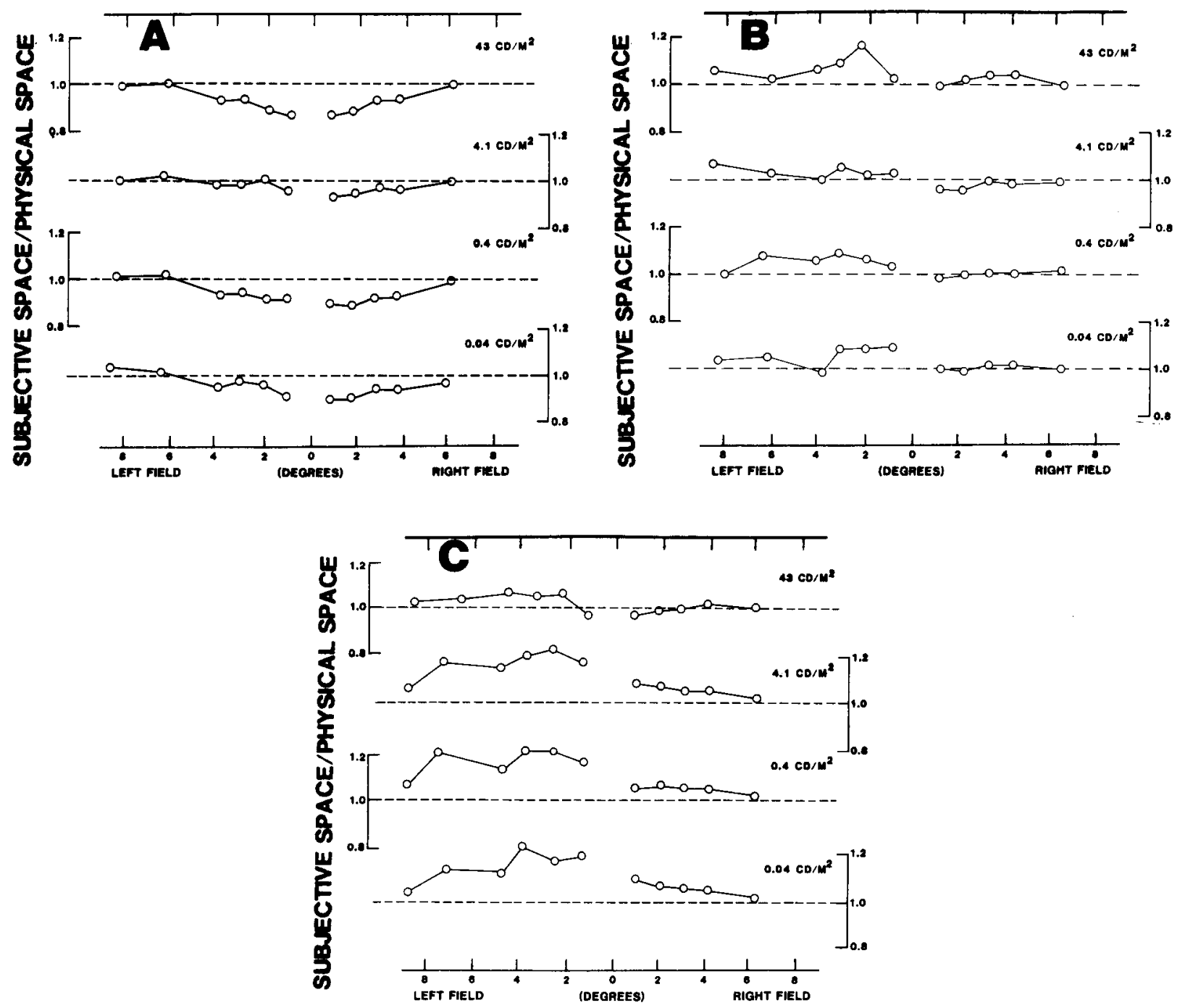

Figure 2. Accuracy of specifying oculocentric direction is represented by a plot of the directions of targets that define subjectively equal fractions of an $8.3^{\circ}$ space in relation to target directions marking physically equal fractions of that space. Standard errors of estimate are equal to or smaller than the plotted points. Panels A, B, and C show data for Observers H.B., R.B., and M.J., respectively. Because the standard space was partitioned successively (using the target-direction resulting from one partitioning as a standard for the next) the inaccuracies represented in the figure are cumulative. Inaccuracy of each individual spatial bisection or match is found by comparison with the inaccuracy of specifying the target(s) serving as standard(s) for that bisection or match. For example, in panel A (Observer H.B. at $43 \mathrm{~cd} / \mathrm{m}^{2}$ ), the direction of the target subjectively defining $1 / 8$ of the $8.3^{\circ}$ right field standard space was 0.86 of $1.04^{\circ}$ (physically $1 / 8$ of $8.3^{\circ}$ ). However, this subjective $1 / 8$ space was obtained by bisecting a space defined by a target at about $2^{\circ}$ in the right field (subjectively $1 / 4$ of the standard space), itself marking only 0.88 of a physical $1 / 4$ of the standard space. Thus, the error in this single bisection (specifying $1 / 8$ of the right field standard space) is equal to $0.88-0.86=2 \%$.

\section{DISCUSSION}

About 30 years ago, Leibowitz et al. reported that observers could discriminate the meridian of the field along which a target is presented equally precisely at and well above the light-detection threshold (Leibowitz et al., 1955a, 1955b). Taken together with our findings-that precision and accuracy of specifying direction along a meridian is not significantly impaired by target luminance reduction-these data indicate that the ability to determine where a target is in the visual field is essentially independent of its luminance. As a result, one would expect that visual functions depending upon information about where a target is in the field (the target's oculocentric direction) would also be unaffected by changes of target luminance. For instance, we have argued and presented evidence previously that the steadiness of fixation is related to the precision of oculocentric visual direction (Bedell et al., 1984); in light of our present findings, it is therefore not surprising that variability of fixation (and other oculomotor behaviors, insofar as they depend on direction information) is little, if at all, affected by reductions of target luminance.

On the other hand, it is tempting to conclude that be- 
Table 1

Precision and Accuracy of Successive Spatial Partitions for Target Luminances Near Absolute Threshold

\begin{tabular}{ccrrr}
\hline & \multicolumn{2}{c}{ Accuracy } & & \multicolumn{2}{c}{ Precision } \\
\cline { 5 - 5 } $\begin{array}{c}\text { Fraction of } 8.3^{\circ} \\
\text { Right-Field Space }\end{array}$ & $\begin{array}{c}\text { (Subjective Space)/ } \\
\text { Physical Space }\end{array}$ & & min arc & percent \\
\hline 3/4 RF & .97 & $7.0 \pm 1.7$ & $1.9 \pm 0.5$ \\
$1 / 2$ RF & .96 & $7.4 \pm 1.9$ & $3.1 \pm 0.8$ \\
$3 / 8$ RF & .94 & $4.5 \pm 1.0$ & $2.5 \pm 0.6$ \\
$1 / 4$ RF & .93 & $2.8 \pm 0.7$ & $2.4 \pm 0.6$ \\
$1 / 8$ RF & .93 & $1.0 \pm 0.2$ & $1.7 \pm 0.4$ \\
$1 / 8$ LF & .92 & $1.0 \pm 0.4$ & $1.8 \pm 0.4$ \\
$1 / 4$ LF & .96 & $3.0 \pm 0.7$ & $2.5 \pm 0.6$ \\
$3 / 8$ LF & .98 & $5.7 \pm 1.4$ & $3.1 \pm 0.8$ \\
$1 / 2$ LF & .96 & $12.6 \pm 3.9$ & $5.2 \pm 1.6$ \\
$3 / 4$ LF & .99 & $18.6 \pm 6.4$ & $5.0 \pm 1.7$ \\
$1 / 1$ LF & .99 & $5.7 \pm 1.3$ & $1.1 \pm 0.3$ \\
\hline
\end{tabular}

haviors that suffer degradation when luminance is reduced depend upon visual information other than or in addition to targets' oculocentric directions. For example, resolution of detail, color discrimination, and contrast sensitivity are impaired substantially as target luminance is reduced (see the introduction) and might reasonably be concluded not to depend primarily upon direction information. Sensitivity to rapid target displacement (Johnson \& Scobey, 1980; Legge \& Campbell, 1981) and to the location of target features in hyperacuity tasks such as the detection of Vernier offset (Baker, 1949; Berry, Riggs, \& Duncan, 1950; Leibowitz, 1955; Westheimer \& McKee, 1977) also are impaired (by a factor of 2 or 3) as luminance is reduced to near threshold values. Although the information mediating displacement sensitivity is unlikely to be a comparison of the target's visual directions (Johnson \& Scobey, 1980; Legge \& Campbell, 1981), it is less clear to what extent direction information mediates performance in hyperacuity tasks in which, depending upon the specific stimulus configuration, any of a variety of suggested cues may operate (Geisler, 1984; Watt, 1984; Watt, Morgan, \& Ward, 1983).

Of particular relevance to this discussion, and in apparent contradiction of our results, are data from a recent study by Klein and Levi (1985). They measured thresholds equal to a few seconds of arc using a three-line bisection task, and found that these hyperacuity thresholds, like those for Vernier offset, worsened approximately twofold as luminance was reduced to just above threshold. Although the bisection task used by Klein and Levi is analogous to ours, a possibly critical difference is that the maximum separation between targets was $5^{\prime}$ of arc. Hence, although it remains possible that information other than oculocentric direction at least partly mediates sensitivity in this and other hyperacuity tasks, it may also be that oculocentric direction information is used, but that its precision is impaired at reduced luminances when targets are very close together.

Consideration of the physiological processes that might underlie oculocentric directionalization suggests why target separation may be critical. It seems likely that the pre- cision with which a target's direction can be specified depends upon how finely the responses to it can be localized on a neural (e.g., cortical) representation, or "map," of the visual field. We speculate that a decreased precision of directionalization at low luminance occurs for targets presented close together in a Vernier or bisection task because responses to the targets overlap on the relevant neural "map." This speculation is predicated on the idea that precise localization of a target's neural activity may be derived by a process of interpolation between the "directions" represented by individual neurons (Barlow, 1981; Crick, Marr, \& Poggio, 1981); such an interpolation process should be hampered if the neural activity associated with individual targets cannot be disambiguated, as when the regions activated by each target overlap. The precision of directionalization could improve with increasing luminance if, thereby, neural activity in this region of overlap became more readily attributable to the individual targets, for instance, by the operation of lateralinhibitory sharpening mechanisms.

On the basis of our results, which are only for targets separated by $1^{\circ}$ and more, we cannot decide whether or not these speculations about neural processing of direction and its role in mediating sensitivity in particular visual tasks are correct. Further experimentation, in which the precision of directionalization is examined as a function of luminance for targets separated by $1^{\circ}$ and less, should help to resolve this issue.

\section{REFERENCES}

ANDREws, D. P., \& Miller, D. T. (1978). Acuity for spatial separation as a function of stimulus size. Vision Research, 18, 615-619.

BAKER, K. E. (1949). Some variables influencing Vemier acuity. Joumal of the Optical Society of America, 39, 567-575.

BARLOW, H. B. (1981). Critical limiting factors in the design of the eye and visual cortex. Proceedings of the Royal Sociery of London, Series B, 212, 1-34.

Bedell, H. E., Barbeito, R., \& Aitsebaomo, P. A. (1984). The precision of oculocentric direction and its role in the stability of fixation. Vision Research, 24, 1157-1161.

Bedell, H. E., \& Flom, M. C. (1983). Normal and abnormal space perception. American Journal of Optometry \& Physiological Optics, 60, 426-435.

Berry, R. N., Riggs, L. A., \& DunCAN, C. P. (1950). The relation of Vernier and depth discriminations to field brightness. Journal of Experimental Psychology, 40, 349-354.

BoyCE, P. R. (1967). The effect of change of target field luminance and colour on fixation eye movements. Optica Acta, 14, 213-217.

CoRnswet, T. N. (1962). The staircase-method in psychophysics. American Journal of Psychology, 75, 485-491.

Crick, F. H. C., Marr, D. C., Poggio, T. (1981). An informationprocessing approach to understanding the visual cortex. In F. $\mathbf{O}$. Schmitt, F. G. Worden, G. Adelman, \& S. G. Dennis (Eds.), The organization of the cerebral cortex (pp. 505-533). Cambridge, MA: MIT Press.

GeISLER, W. S. (1984). Physical limits of acuity and hyperacuity. Joumal of the Optical Society of America, Part A, 1, 775-782.

Helmholtz, H., von (1924/1925). Treatise on physiological optics (Vol. 2, pp. 369ff; J. P. C. Southall, Ed.). Menasha, WI: Optical Society of America.

HeLMHOLTZ, H., vON (1925). Treatise on physiological optics (Vol. 3, pp. 168ff; J. P. Southall, Ed.). Menasha, WI: Optical Society of America. 
Johnson, C. A., \& Scobey, R. P. (1980). Foveal and peripheral displacement thresholds as a function of stimulus luminance, line length and duration of motion. Vision Research, 20, 709-715.

KELLY, D. A. (1961). Visual responses to time dependent stimuli-I. Amplitude sensitivity measurements. Journal of the Optical Society of America, 51, 422-429.

KLEIN, S. A., \& LEVI, D. M. (1985). Hyperacuity thresholds of $1 \mathrm{sec}$ : Quantitative predictions and empirical validation. Journal of the Optical Society of America, Part A, 2, 1170-1190.

LeGge, G. E., \& CAMPBELL, F. W. (1981). Displacement detection in human vision. Vision Research, 21, 205-213.

LEIBOWITZ, H. W. (1955). Some factors influencing the variability of Vernier adjustments. American Journal of Psychology, 68, 266-273.

Leibowitz, H. W., Myers, N. A., \& Grant, D. A. (1955a). Frequency of seeing and radial localization of single and multiple visual stimuli. Journal of Experimental Psychology, 50, 369-373.

Leibowitz, H. W., Myers, N. A., \& Grant, D. A. (1955b). Radial localization of a single stimulus as a function of luminance and duration of exposure. Journal of the Optical Society of America, 45, 76-78.

LEVI, D. M., \& KleIN, S. A. (1983). Spatial localization in normal and amblyopic vision. Vision Research, 23, 1005-1017.

OGLE, K. N. (1932). An analytical treatment of the longitudinal horopter; its measurement and application to related phenomena, especially to the relative size and shape of the ocular images. Journal of the Optical Society of America, 22, 665-728.

SCHLAER, S. (1937). The relation between visual acuity and illumination. Journal of General Physiology, 21, 165-188.

Steinman, R. M. (1965). Effect of target size, luminance, and color on monocular fixation. Journal of the Optical Society of America, $\mathbf{5 5}$, 1158-1165.

Stevens, H. C., \& Ducasse, C. J. (1912). The retina and righthandedness. Psychological Review, 19, 1-31.

VAlCIUKAS, J. A. (1972). Optokinetic thresholds in the normal monkey. Vision Research, 12, 1397-1407.
VAN NES, F. L., \& Bouman, M. A. (1967). Spatial modulation transfer in the human eye. Journal of the Optical Society of America, 57, 401-406.

WATT, R. J. (1984). Towards a general theory of the visual acuities for shape and spatial arrangement. Vision Research, 24, 1377-1386.

Watt, R. J., Morgan, M. J., \& Ward, R. M. (1983). The use of different cues in Vernier acuity. Vision Research, 23, 991-995.

WEALE, R. A. (1951). Hue-discrimination in para-central parts of the human retina measured at different luminance levels. Journal of Physiology, 113, 115-122.

Westheimer, G. , \& MCKeE, S. P. (1977). Integration regions for visual hyperacuity. Vision Research, 17, 89-93.

Winterson, B. J., \& Steinman, R. M. (1978). The effect of luminance on human smooth pursuit of peripheral and foveal targets. Vision Research, 18, 1165-1172.

\section{NOTES}

1. A control condition, in which the standard spatial extent was defined by a target in the left field, showed that differences in precision were associated primarily with differences between matching and bisecting, rather than with differences between the nasal and temporal visual fields.

2. That normal observers make small constant errors in matching the directions of monocularly viewed targets in the right and left fields is well documented (for reviews, see Helmholtz, 1925, and Bedell \& Flom, 1983). Note that our procedure of successive bisection reveals that observers also make idiosyncratic constant errors in specifying equally spaced directions within the right and left hemifields.

(Manuscript received January 8, 1985; revision accepted for publication August 2, 1985.) 